\title{
Sharp Total Variation Bounds for Finitely Exchangeable Arrays
}

\author{
Alexander Volfovsky* \\ Statistics Department, Harvard University, Cambridge, MA, USA \\ Edoardo M. Airoldi \\ Statistics Department, Harvard University, Cambridge, MA, USA
}

\begin{abstract}
In this article we demonstrate the relationship between finitely exchangeable arrays and finitely exchangeable sequences. We then derive sharp bounds on the total variation distance between distributions of finitely and infinitely exchangeable arrays.

Keywords: Finite exchangeability, arrays, total variation bound, networks, hypothesis testing
\end{abstract}

\section{Introduction}

The study of invariance properties of probability distributions has long been the focus of statisticians and probabilists (De Moivre, 1756; de Laplace, 1820; De Finetti, 1931; Hewitt and Savage, 1955; Aldous, 1985; Austin and Panchenko, 2013). The most common type of invariance is the notion of indepe ndent ind identicaliy distribibuted dirstributions. It is rundam ntai ifor ciass cai resuits such as the weak and strong laws of large numbers and central limit theorems. Relaxation of the statistical notion of independence in this definition to simply requiring that the order of a sequence of random variables not affect their joint distribution gives rise to the notion of exchangeable random variable sequences. These variables are marginally identically distributed but they can exhibit different forms of dependence. Infinite exchangeability of a sequence

\footnotetext{
* Corresponding author

Email addresses: vol fovsky@fas. harvard.edu (Alexander Volfovsky), airoldi@fas.harvard.edu (Edoardo M. Airoldi)
}

Preprint submitted to Statistics and Probability Letters

February 20, 2016 
of random variables is in turn related back to independent and identically distributed random variable sequences via a famous theorem due to de Finetti (De Finetti, 1931, 1972). Considering an infinitely exchangeable sequence $X_{1}, X_{2}, \ldots$ of binary random variables the theorem states that there is a unique probability measure $\mu$ such that for all $n \geq 1$

$$
\operatorname{Pr}\left(X_{1}=e_{1}, \ldots, X_{n}=e_{n}\right)=\int p^{\sum e_{i}}(1-p)^{n-\sum e_{i}} d \mu(p) .
$$

Generalizations of this famous theorem to higher dimensional arrays were given independently by Hoover (1989) and Aldous (1981): For example consider an infinitely row-column weakly exchangeable binary matrix $X$. The distribution of such a matrix is invariant under the permutation of the row and column indices of the matrix by the same permutation. Intuitively, such a matrix can represent relational data, where the shared index set of the rows and columns represents an actor, while the entries of the matrix represent the existence of a relationship among the actors. The Aldous-Hoover representation for this matrix is

$$
X_{i j}=\mathbf{1}\left[W\left(U_{i}, U_{j}\right) \geq \lambda_{i j}\right]
$$

where $U_{i}$ and $\lambda_{i j}$ are independent uniform $(0,1)$ random variables and $W$ is a measurable symmetric function from $[0,1]^{2} \rightarrow[0,1]$. Intuitively, the $U_{i}$ can be interpreted as actor attributes while the $\lambda_{i j}$ as pairwise attributes.

These results have served as foundation for many Bayesian methods. For example, proofs of the consistency of Bayesian procedures rely on the assumption that the data is infinitely exchangeable, that is conditionally i.i.d (Berk, 1970). Similarly, the analysis of relational data heavily relies on the Aldous-Hoover representation for arrays ( $\overline{\mathrm{Hoff}}$ et al. 2002). The power of these results is derived from the assumption that the index set of the array is infinite. Specifically, it is well known that de Finetti's theorem does not hold for finitely exchangeable sequences (Diaconis, 1977; Diaconis and Freedman, 1980). A similar result has been known, though apparently not written down, for the failure of the Aldous-Hoover representation to hold for finitely row-column exchangeable arrays. Consider the following distribution on $2 \times 2$ binary matrices: $P\left(x_{11}=x_{22}=0\right)=1, P\left(x_{12}=x_{21}\right)=0$ and $P\left(x_{12}=1\right)=P\left(x_{21}=1\right)=1 / 2$. 
This is clearly a jointly row-column exchangeable distribution but an Aldous-Hoover representation would require:

$$
\begin{aligned}
& 0=P\left(x_{12}=0, x_{21}=0\right)=\int(1-p)^{2} d \mu(p) \\
& 0=P\left(x_{12}=1, x_{21}=1\right)=\int p^{2} d \mu(p)
\end{aligned}
$$

5 for $\mu$ a mixing measure as in the de Finetti representation in Eq (1).

Notions of finite invariance described above have received a lot of attention in the case of sequences, but much less so for networks and general arrays. Specifically, our work is motivated by the pervasiveness of the assumption of joint row-column exchangeability throughout the field of network analysis (Holland et al., 1983; Hoff et al.,

10 2002, Diaconis and Janson, 2007; Airoldi et al., 2009, Bickel and Chen, 2009). While certain network data have the potential representation as a sample from an infinite population, it is very common to observe the whole finite network such as in the study of trade between countries (Volfovsky and Hoff, 2014), friendship networks among students in the same class (Hoff et al. 2013) and interactions among monks in a monastery (Sampson, 1969). In these cases an assumption of exchangeability of the nodes is still desirable as there is no information in the individual labels, but the assumption of infinite exchangeability is inappropriate since there is no infinite network the data could have been sampled from.

This article is motivated by these finitely exchangeable network scenarios and is not intended as a review of infinite exchangeability. A comprehensive overview of the assumptions and representations of infinite exchangeability for networks and general arrays is available in Orbanz and Roy (2013). In the next section we derive sharp bounds on the total variation distance between finitely exchangeable network distributions and their infinitely exchangeable extensions. This is done by relating the distribution of the singular value decomposition of a relational matrix to the distribution of finitely exchangeable sequences. Section 3 extends these results to general $k$ dimensional arrays where each dimension is independently exchangeable. To arrive at this result we extend the results of Freedman (1977) to bounds on the total variation distance between sampling with and without replacement from multiple urns. The desire for an exact 
bound was posed as open problem 15.10 by Aldous (1985, p. 137). In the Discussion we provide an explicit statement of a representation theorem for distributions that are invariant under the operations of a finite group $G$. This representation can be used to compute a $G$-invariant non-parametric maximum likelihood estimate of a distribution as well as for the construction of test statistics for the infinite exchangeability of a 35 distribution.

\section{Sharp total variation bounds for networks}

In this section we derive bounds for the total variation distance between the distributions of finitely and infinitely exchangeable networks. This quantitative summary provides insight into the efficacy of the assumption of infinite exchangeability. By taking limits of the bounds in the number of nodes in the network we recover the classical Aldous-Hoover representation. Recall that a distribution of a network with $\mathrm{m}$ nodes is exchangeable, that is its representation as a square relational data matrix $X$ is jointly row-column exchangeable, if for all $g \in S_{m}$ (= symmetric group) we have $\operatorname{Pr}(g X=A)=\operatorname{Pr}(X=A)$ where $g$ acts on the rows and columns of $X$ simul-

45 taneously. The results of Aldous (1981) and Hoover (1979) provide a representation theorem for infinitely exchangeable networks in the spirit of de Finetti's theorem for infinitely exchangeable sequences: If $\left(X_{i j}\right)_{1,1}^{\infty, \infty}$ is an infinitely exchangeable network then it can be written as $X_{i j}=f\left(\alpha, u_{i}, u_{j}, \lambda_{i j}\right)$ for a measurable function $f$ that is symmetric in its middle arguments and $\alpha, u_{i}, \lambda_{i j}$ all independent uniform $(0,1)$. This is explicitly called joint or weak exchangeability. This representation is frequently used when describing relational data where the object of interest is a square array $X$.

It is natural to ask how close the finitely exchangeable distributions are to the Aldous-Hoover representation of an infinitely row-column exchangeable array. The example of the failure of the Aldous-Hoover representation for jointly exchangeable arrays is similar in spirit to the classical example provided by Diaconis (1977) for the failure of the de Finetti's theorem for a finite sequence. We first present the sharp total variation bound for finitely exchangeable sequences found in Diaconis and Freedman (1980) and then employ a singular value decomposition to extend the result to 
networks.

60 Theorem 1 (Diaconis and Freedman (1980) Theorem 13). Let $(\mathcal{S}, \mathcal{B})$ be an abstract measurable space and let $\mathcal{S}^{\star}$ be the set of probabilities on $(\mathcal{S}, \mathcal{B})$ endowed with the smallest $\sigma$-field $\mathcal{B}^{\star}$ that makes $p \rightarrow p(A)$ measurable for all $A \in \mathcal{B}$. Let $P$ be an exchangeable probability on $\left(\mathcal{S}^{n}, \mathcal{B}^{n}\right), P_{k}$ be the projection of $P$ onto $\left(\mathcal{S}^{k}, \mathcal{B}^{k}\right)$. There exists a probability $\mu$ on $\left(\mathcal{S}^{\star}, \mathcal{B}^{\star}\right)$ such that if $P_{\mu k}$ is a projection of an infinitely ex-

65 changeable distribution onto $\left(\mathcal{S}^{k}, \mathcal{B}^{k}\right)$ with mixing measure $\mu$, then $\left\|P_{k}-P_{\mu k}\right\| \leq$ $2 \beta(k, n)$ for all $k \leq n$ where $\beta(k, n)=1-n^{-k} n ! /(n-k) !$.

Proof outline. This result is a direct consequence of finding the total variation bounds for sampling with and without replacement from $\mathcal{S}^{k}$. Sampling without replacement represents an extreme point of the space of exchangeable distributions while sampling with replacement is a power probability. By letting $\mu$ be the distribution of the empirical measure implied by $P$, the difference $P_{k}(A)-P_{\mu k}(A)$ (for $A \in \mathcal{S}^{k}$ ) can be written as a mixture of differences between sampling without and with replacement. The sharpness of the bound is immediate as it is achieved by the distance between sampling with and without replacement from an unconstrained statespace $\mathcal{S}$.

75 The projection onto $\left(\mathcal{S}^{k}, \mathcal{B}^{k}\right)$ describes the notion of extendibility of an exchangeable distribution. That is, the theorem requires that an exchangeable distribution on $\left(\mathcal{S}^{k}, \mathcal{B}^{k}\right)$ be extendible to an exchangeable distribution on $\left(\mathcal{S}^{n}, \mathcal{B}^{n}\right)$ as such $P_{k}$ is a marginal distribution of $P$. A similar construct is available for networks as well: an exchangeable distribution on an $m$ dimensional network is extendible to $r$ dimensions

80 if it is the marginal of an $r$ dimensional exchangeable distribution on networks. Infinite extendibility is equivalent to infinite exchangeability and so finite extendibility is a weaker assumption.

Theorem 2 (Total variation of jointly row-column exchangeable arrays). Finitely joint row-column ( $m$ dimensional) exchangeable (and $r$ extendible) probabilities are bounded in total variation distance from infinitely joint row-column exchangeable probabilities by $2 \beta(m, r)$. This bound is sharp. 
Proof. We first demonstrate that the distribution of $X$ is jointly row-column exchangeable if and only if the distribution of its left and right eigenvectors is row exchangeable. Since the singular value decomposition is not unique, care must be taken. First, we overload notation to write the singular value decomposition (SVD) of $X$ as $X \stackrel{d}{=}$ $U(X) D(X) V(X)^{t}$. We choose a decomposition such that $D(X)$ is a diagonal matrix with non-negative entries in descending order. The functions $U(\cdot)$ and $V(\cdot)$ map to a pre-specified choice of left and right singular vectors of $X$ (for example, for nondegenerate singular values one can use the SignFlip function of Bro et al. (2008)). For null singular values, the corresponding columns of $U(X)$ and $V(X)$ are distributed uniformly over the bases for the left and right null spaces of $X$. Define a set $E(X)=\left\{O\right.$ orthogonal : $\left.D(X)=O D(X) O^{t}\right\}$, the space of orthogonal matrices that leaves the diagonal singular value matrix unchanged. For example: If all the singular values are unique then $E(X)$ contains all diagonal matrices with entries \pm 1 . If the first two singular value are the same then $E(X)$ also contains orthogonal matrices for which the leading $2 \times 2$ submatrix is an orthogonal matrix. Now, let the random variable $S(X)$ be uniformly distributed on $E(X)$ and define the random variables

$$
\tilde{U}(X) \stackrel{d}{=} U(X) S(X), \tilde{V}(X) \stackrel{d}{=} V(X) S(X) .
$$

These variables define random rotations of the left and right singular vectors of $X$. This mechanism of introducing additional randomness for identifying distributions of eigenvectors was previously used in (Tao and $\mathrm{Vu}, 2011$ ). Since $S(X)$ is uniformly distributed on $E(X)$, it is clear that $\tilde{U}(X)$ and $\tilde{V}(X)$ are uniformly distributed on the equivalence classes

$$
\operatorname{eqc}(U(X))=\{U(X) O: O \in E(X)\}, \operatorname{eqc}(V(X))=\{V(X) O: O \in E(X)\} .
$$

Let $g$ be an element of the permutation group and define its action on $X$ as the permutation of the rows and columns jointly and its action on the singular vectors as the permutation of the rows only. Since eqc $(U(g X))=g \operatorname{eqc}(U(X))$ and eqc $(V(g X))=$ 9o $\quad g$ eqc $(V(X))$ we have that $g X \stackrel{d}{=} X$ implies $g[\tilde{U}(X) ; \tilde{V}(X)] \stackrel{d}{=}[\tilde{U}(X) ; \tilde{V}(X)]$. We have thus shown that if $X$ is row-column exchangeable then $[\tilde{U}(X) ; \tilde{V}(X)]$ is rowexchangeable. 
If the right and left eigenvectors are row exchangeable then $X$ is jointly row-column exchangeable by construction. To apply Theorem 1 we require extendibility of a sequence. To understand extendibility we note that if an $r \times r$ matrix is row-column exchangeable then the an $m \times m$ submatrix is also row-column exchangeable. Defining the $U$ and $V$ above be the $m \times r$ rows of the singular value decomposition of the large $r \times r$ matrix the above results hold. Constructing the relevant $[\tilde{U}, \tilde{V}]$ matrix and treating the rows as a sequence we see that we can directly apply Theorem 1 to get the desired result.

The theorem translates the discussion of the joint row-column exchangeability of a distribution on square matrices to the discussion of the joint row exchangeability of orthogonal matrices.

\section{Sharp total variation bounds for general arrays}

Without loss of generality, in this section we prove theorems for two dimensional arrays and then provide an outline of how one extends the results to higher dimensions. Recall that a distribution of a $m \times n$ array $X$ is row-column exchangeable if for all $\left(g_{1}, g_{2}\right) \in S_{m} \times S_{n}$ we have $\operatorname{Pr}\left(\left(g_{1}, g_{2}\right) X=A\right)=\operatorname{Pr}(X=A)$ where $g_{1}$ acts on the rows and $g_{2}$ acts on the columns of $X$. The Aldous-Hoover representation for an infinitely row-column exchangeable array is: $\left(X_{i j}\right)_{1,1}^{\infty, \infty}$ is infinitely row-column exchangeable then $X_{i j}=f\left(\alpha, u_{i}, v_{j}, \lambda_{i j}\right)$ for a measurable function $f$ and $\alpha, u_{i}, v_{j}, \lambda_{i j}$ all independent uniform $(0,1)$ random variables. To find the total variation bounds for general exchangeable arrays we require the following extension of Theorem 1 ;

Theorem 3 (Total variation bounds for partial exchangeability). Let $\left(\mathcal{S}_{1}, \mathcal{B}_{1}\right),\left(\mathcal{S}_{2}, \mathcal{B}_{2}\right)$ be abstract measurable spaces and let $\mathcal{S}_{i}^{\star}$ be the set of probabilities on $\left(\mathcal{S}_{i}, \mathcal{B}_{i}\right)$ endowed with the smallest $\sigma$-field $\mathcal{B}_{i}^{\star}$ that makes $p \rightarrow p(A)$ measurable for all $A \in \mathcal{B}_{i}$. Let $P$ be a partially exchangeable probability on $\left(\mathcal{S}_{1}^{n_{1}} \times \mathcal{S}_{2}^{n_{2}}, \mathcal{B}_{1}^{n_{1}} \times \mathcal{B}_{2}^{n_{2}}\right), P_{k_{1}, k_{2}}$ be the projection of $P$ onto $\left(\mathcal{S}_{1}^{k_{1}} \times \mathcal{S}_{2}^{k_{2}}, \mathcal{B}_{1}^{k_{1}} \times \mathcal{B}_{2}^{k_{2}}\right)$. Then there exists a probability $\mu$ on $\left(\mathcal{S}_{1}^{\star} \times \mathcal{S}_{2}^{\star}, \mathcal{B}_{1}^{\star} \times \mathcal{B}_{2}^{\star}\right)$ such that if $P_{\mu k_{1}, k_{2}}$ is a projection of an infinitely partially 
exchangeable distribution onto $\left(\mathcal{S}_{1}^{k_{1}} \times \mathcal{S}_{2}^{k_{2}}, \mathcal{B}_{1}^{k_{1}} \times \mathcal{B}_{2}^{k_{2}}\right)$ with mixing measure $\mu$, then

$$
\left\|P_{k_{1}, k_{2}}-P_{\mu k_{1}, k_{2}}\right\| \leq 2 \beta\left(\left(k_{1}, k_{2}\right),\left(n_{1}, n_{2}\right)\right) \forall k_{1} \leq n_{1}, k_{2} \leq n_{2}
$$

where

$$
\beta\left(\left(k_{1}, k_{2}\right),\left(n_{1}, n_{2}\right)\right)=1-n_{1}^{-k_{1}} n_{1} ! /\left(n_{1}-k_{1}\right) ! n_{2}^{-k_{2}} n_{2} ! /\left(n_{2}-k_{2}\right) ! .
$$

This bound is tight.

Proof. The proof is the same as the proof of Theorem 1 after making the obvious substitutions. Specifically, in Theorem 1 we employed results on sampling from an urn that included all possible sequences of length $n$ composed of elements of a set $\mathcal{S}$. Here we consider sampling from a pair of urns that together include all possible pairs of sequences of length $n_{1}+n_{2}$ where the first sequence is composed of elements of $\mathcal{S}_{1}$ while the second of elements of $\mathcal{S}_{2}$. The distributions we compare are sampling with and without replacement from this collection of urns. Any finitely partially exchangeable distributions will be a mixture of the distributions of sampling without replacement from these urns while the projection of infinitely partially exchangeable distributions will be a mixture of the distributions of sampling with replacement from these urns. The bound and the tightness of the bound are given by the technical lemmas in the Appendix.

We now extend Theorem 2 to separately exchangeable arrays.

Theorem 4 (Total variation bounds). Let $P_{m, n}^{r, q}$ be a distribution on $m \times n$ dimensional matrices that is finitely row-column exchangeable and is extendible to a row-column exchangeable distribution on $r \times q$ dimensional matrices. Let $P_{\mu, m, n}$ be the projection of an infinitely row-column exchangeable distribution onto the space of $m \times n$ dimensional matrices. Then for $\|\cdot\|$ representing the total variation norm we have

$$
\left\|P_{m, n}^{r, q}-P_{\mu, m, n}\right\| \leq \beta((m, n),(r, q)) .
$$

The bound is tight. 
Proof. The proof is similar to that of Theorem 2 The singular value decomposition of the matrix $X \stackrel{d}{=} U(X) D(X) V(X)^{t}$ is again considered, but the separate exchangeability of the rows and columns implies that the rows of the left and right eigenvector matrices are separately permuted. The random variables $S(X), \tilde{U}(X), \tilde{V}(X)$ are constructed as in Theorem 2. By concatenating $\tilde{U}(X)$ and $\tilde{V}(X)$ along the rows into $Z=\left[\tilde{U}(X)^{t} ; \tilde{V}(X)^{t}\right]^{t}$, a $m+n$ row matrix, we see that separate row column exchangeability of $X$ is equivalent to the partial exchangeability of the rows of $Z$. The results then follows from Theorem 3 .

The contribution of Theorem 4 is two-fold. As far as we know, this is the first time an explicit form for the total variation bound for row-column exchangeability has been proposed. More importantly, we now see that the rate of convergence of the total variation bound is bounded by $\left(m^{2}-m\right) / q+\left(n^{2}-n\right) / r$ for matrices, similar to the results of Diaconis and Freedman (1980) for sequences. Knowing this bound provides a justification for the assumption of infinite row-column exchangeability (allowing for an Aldous-Hoover representation) in high-dimensional statistics when both the subjects and features are not independent but can assumed to be exchangeable and network analysis where the labels of the nodes do not carry information.

To extend the results to higher dimensions we require a generalization of the singular value decomposition. For example, we can consider the Higher Order Singular Value Decomposition of Kolda and Bader (2009): A $k$-dimensional array with dimensions $n_{1}, \ldots, n_{k}$ can be decomposed via the Tucker decomposition into a core array $S$ and $k$ orthogonal matrices $U_{1}, \ldots, U_{k}$. A distribution over arrays that is invariant under independent permutations of the index sets of the different dimensions of the array is equivalent to a distribution over arrays that is invariant under independent permutations of the rows of the orthogonal matrices $U_{1}, \ldots, U_{k}$. Lemma 6 of Appendix A provides a $k$-dimensional version of the bound in Theorem 3 and the proof of the sharpness of the bound follows with the obvious changes. 


\section{Discussion}

In this paper we have demonstrated sharp bounds for the total variation distance between finitely and infinitely exchangeable distributions for networks and for general arrays. These results answer an open question of Aldous (1985). Addressing the original motivation behind the paper, the tight bound for the jointly row-column exchangeable distributions provides an insight into the efficacy of the assumption of existence of an Aldous-Hoover representation when analyzing relational data. Similar results for sequences were previously used to justify the use of de Finetti's theorem for Bayesian causal inference (Rubin, 1978). The magnitude of the deviation of parameter estimates for the finite exchangeability-infinite exchangeability paradigm is a part of ongoing research by the authors. We conjecture that any estimator that has a faster rate of convergence than the total variation bounds should have similar properties whether one assumes finite or infinite exchangeability. This is motivated empirically by the successful use of infinitely exchangeable methods when the true generating process cannot be infinitely exchangeable as is the case of Sampson's monastery (Sampson, 1969) and high school classrooms (Hoff et al., 2013). Success is measured by the external validation of the results by subject matter scientists.

The results of the paper require an assumption of extendibility of the networks and arrays in question. While we believe this to be a more reasonable assumption than infinite exchangeability, it might fail to hold in some situations. When this is the case inference that does not take into account the finite exchangeability of the data generating distribution is prone to the errors described in the introduction. To accommodate this scenario we present a general characterization theorem for distributions that are invariant under finite group operations.

Theorem 5 (Characterization Theorem). Let $G$ be a finite group acting on a finite state space $\mathcal{X}$. The collection of $G$-invariant measures on $\mathcal{X}$ is a convex set with extreme points $e_{G(x)}$ indexed by the orbits of $\mathcal{X}$ and placing weight $1 /|G(x)|$ on each member of $G(x)$ and 0 everywhere else.

Proof. Convexity is a trivial consequence of the convexity of the general space of probability distributions on $\mathcal{X}$ when $|\mathcal{X}|<\infty$. First, if a distribution is $G$-invariant then 
the probability of two elements within an orbit must be equal and so it is constant on the orbits. Conversely, if a distribution is uniform over a single orbit then it is necessarily invariant under the group operation. To see that these $e_{G(x)}$ are extreme we note that they all have disjoint support by definition and so cannot be written as convex the null distributions can be constructed via Monte Carlo simulation.

\section{Acknowledgments}

We would like to thank the editor, associate editor and two reviewers for detailed comments that have helped improve this manuscript. This work was supported, in part, by

ONR award N00014-14-1-0485, by ARO MURI award W911NF-11-1-0036 and NSF CAREER grant IIS-1149662 to Harvard University. AV is an NSF MSPRF on NSF DMS-1402235. EMA is a Sloan Research Fellow. 


\section{Appendix A. Technical lemmas}

To prove Theorem 3 we require an extension of the results of Freedman (1977) to problems with multiple urns. We follow Freedman's notation and method. In particular, consider a collection of $d$ urns $F_{1}, \ldots, F_{d}$ where $F_{i}$ contains elements $\left\{f_{i 1}, \ldots, f_{i n_{i}}\right\}$. Let $F_{i}^{k_{i}}$ consist of the $k_{i}$-tuples of elements of $F_{i}$. Sampling with replacement from the collection of $d$ urns induces a uniform probability $M$ on $F_{1}^{k_{1}} \times$ $\cdots \times F_{d}^{k_{d}}$ where

$$
M\left(\left\{s_{11}, \ldots, s_{1 k_{1}}\right\}, \ldots,\left\{s_{d 1}, \ldots, s_{d k_{d}}\right\}\right)=\frac{1}{n_{1}^{k_{1}} \cdots n_{d}^{k_{d}}}
$$

Letting $B$ be the vectors $\left\{s_{11}, \ldots, s_{1 k_{1}}\right\}, \ldots,\left\{s_{d 1}, \ldots, s_{d k_{d}}\right\} \in F_{1}^{k_{1}} \times \cdots \times F_{d}^{k_{d}}$ for which all the components are unequal, sampling without replacement is given by

$$
Q\left(\left\{s_{11}, \ldots, s_{1 k_{1}}\right\}, \ldots,\left\{s_{d 1}, \ldots, s_{d k_{d}}\right\}\right)=\frac{\left(n_{1}-k_{1}\right) ! \cdots\left(n_{d}-k_{d}\right) !}{n_{1} ! \cdots n_{d} !} .
$$

Lemma 6 (Sampling with and without replacement). The total variation distance between sampling with and without replacement from multiple urns is

$$
\|M-Q\|=1-\frac{n_{1} ! \cdots n_{d} !}{\left(n_{1}-k_{1}\right) ! \cdots\left(n_{d}-k_{d}\right) ! n_{1}^{k_{1}} \cdots n_{d}^{k_{d}}} .
$$

Proof. The proof is by construction. We note that $Q(B)=1$ and the total variation norm is given by $Q(B)-M(B)$. To calculate $M(B)$ we note that the probability of any particular sequence is equal under $M$ and so we simply need to count the number of sequences in $B$. This is an easy combinatorial exercise and each urn has $\frac{n_{i} !}{\left(n_{i}-k_{i}\right) !}$ sequences of length $k_{i}$ with unique entries. Thus $M(B)=\frac{n_{1} ! \cdots n_{d} !}{\left(n_{1}-k_{1}\right) ! \cdots\left(n_{d}-k_{d}\right) ! n_{1}^{k_{1}} \cdots n_{d}^{k_{d}}}$ and the lemma follows.

Lemma 7 (Tight bounds). The bound in Theorem 3 is tight. That is

$$
\left\|P_{n_{1}, n_{2}, k_{1}, k_{2}}-P_{\mu, k_{1}, k_{2}}\right\| \geq\left\|P_{n_{1}, n_{2}, k_{1}, k_{2}}-M\right\|=1-\frac{n_{1} ! n_{2} !}{\left(n_{1}-k_{1}\right) !\left(n_{2}-k_{2}\right) ! n_{1}^{k_{1}} n_{2}^{k_{2}}}
$$

where $M$ is as in Lemma 6 .

Proof. Without loss of generality we let the distributions of interest be over two urns with balls labeled $1^{1}, \ldots, n_{1}^{1}$ and $1^{2}, \ldots, n_{2}^{2}$ respectively. We note that the equality 
in the statement is given by Lemma 6 Thus as in the original proof of tightness in Diaconis and Freedman (1980) we must show that for $B$ as defined in Lemma 6 we have $\left(p_{1}^{k_{1}} p_{2}^{k_{2}}\right)(B) \leq M(B)$ for any distributions $p_{1}, p_{2}$ on the the sets $\left\{1^{1}, \ldots, n_{1}^{1}\right\}$ and $\left\{1^{2}, \ldots, n_{2}^{2}\right\}$ respectively. To see that this is true we note $M$ is a products of two pure power probabilities and that we can write the set $B=B_{1} \times B_{2}$ where $B_{1}$ are all the sets of length $k_{1}$ of unique elements in the first urn and similarly for $B_{2}$. As in Diaconis and Freedman (1980) we note that the Schur convexity of the indicator functions $1_{B_{1}}$

230 and $1_{B_{2}}$ implies that $p_{i}^{k_{i}}\left(B_{i}\right) \leq M_{i}\left(B_{i}\right)$. This implies that $P_{\mu, k_{1}, k_{2}}(B) \leq M(B)$ as desired.

\section{References}

A. De Moivre, The doctrine of chances: or, A method of calculating the probabilities of events in play, vol. 1, Chelsea Publishing Company, 1756.

P. S. de Laplace, Théorie analytique des probabilités, Courcier, 1820.

B. De Finetti, Funzione caratteristica di un fenomeno aleatorio .

E. Hewitt, L. J. Savage, Symmetric measures on Cartesian products, Transactions of the American Mathematical Society (1955) 470-501.

D. Aldous, Exchangeability and related topics, École d'Été de Probabilités de SaintFlour XIII?1983 (1985) 1-198.

T. Austin, D. Panchenko, A hierarchical version of the de Finetti and Aldous-Hoover representations, Probability Theory and Related Fields (2013) 1-15.

B. De Finetti, Probability, induction and statistics, vol. 8, Wiley New York, 1972.

D. Hoover, Tail fields of partially exchangeable arrays, Journal of Multivariate Analysis 31 (1) (1989) 160-163.

D. J. Aldous, Representations for partially exchangeable arrays of random variables, Journal of Multivariate Analysis 11 (4) (1981) 581-598. 
R. H. Berk, Consistency a posteriori, The Annals of Mathematical Statistics (1970) 894-906.

P. D. Hoff, A. E. Raftery, M. S. Handcock, Latent space approaches to social network analysis, Journal of the american Statistical association 97 (460) (2002) 1090-1098.

P. Diaconis, Finite forms of de Finetti's theorem on exchangeability, Synthese 36 (2) (1977) 271-281.

P. Diaconis, D. Freedman, Finite exchangeable sequences, The Annals of Probability (1980) 745-764.

P. W. Holland, K. B. Laskey, S. Leinhardt, Stochastic blockmodels: First steps, Social networks 5 (2) (1983) 109-137.

P. Diaconis, S. Janson, Graph limits and exchangeable random graphs, arXiv:0712.2749 .

E. Airoldi, D. Blei, S. Fienberg, E. Xing, Mixed membership stochastic blockmodels, in: Advances in Neural Information Processing Systems, 33-40, 2009.

P. J. Bickel, A. Chen, A nonparametric view of network models and Newman-Girvan and other modularities, Proceedings of the National Academy of Sciences 106 (50) (2009) 21068-21073.

265 A. Volfovsky, P. D. Hoff, Testing for nodal dependence in relational data matrices, Journal of the American Statistical Association (just-accepted) (2014) 00-00.

P. Hoff, B. Fosdick, A. Volfovsky, K. Stovel, Likelihoods for fixed rank nomination networks, Network Science 1 (03) (2013) 253-277.

S. F. Sampson, Crisis in a cloister, Ph.D. thesis, Cornell University, Ithaca, 1969.

P. Orbanz, D. M. Roy, Bayesian Models of Graphs, Arrays and Other Exchangeable Random Structures, arXiv preprint arXiv:1312.7857 .

D. Freedman, A remark on the difference between sampling with and without replacement, Journal of the American Statistical Association 72 (359) (1977) 681. 
D. N. Hoover, Relations on probability spaces and arrays of random variables, Preprint, Institute for Advanced Study, Princeton, NJ 2.

R. Bro, E. Acar, T. G. Kolda, Resolving the sign ambiguity in the singular value decomposition, Journal of Chemometrics 22 (2) (2008) 135-140.

T. Tao, V. Vu, Random matrices: universality of eigenvectors, Tech. Rep., 2011.

T. G. Kolda, B. W. Bader, Tensor decompositions and applications, SIAM review 51 (3) (2009) 455-500.

D. B. Rubin, Bayesian inference for causal effects: The role of randomization, The Annals of Statistics (1978) 34-58.

R. A. Wijsman, et al., Random orthogonal transformations and their use in some classical distribution problems in multivariate analysis, The Annals of Mathematical Statistics 28 (2) (1957) 415-423.

M. L. Eaton, Group invariance applications in statistics, in: Regional conference series in Probability and Statistics, JSTOR, i-133, 1989. 